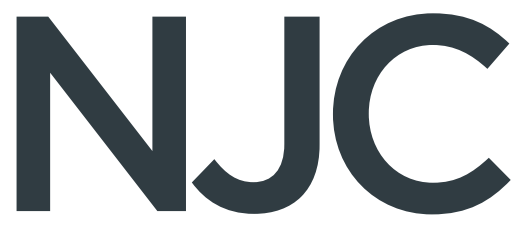

A journal for new directions in chemistry

New Journal of Chemistry

rsc.li/njc

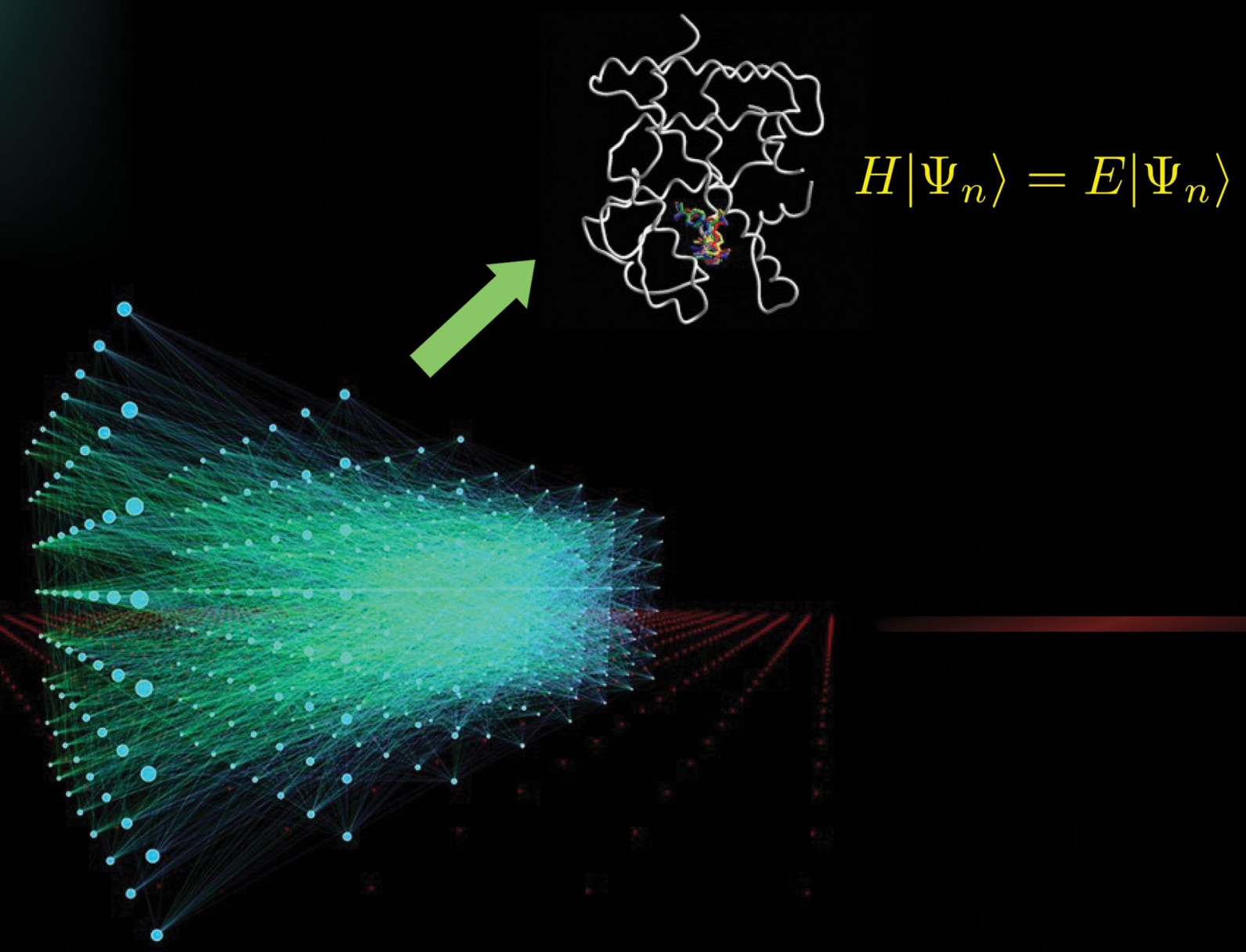


Check for updates

Cite this: New J. Chem., 2020, 44, 20914

Received 22nd May 2020,

Accepted 7th November 2020

DOI: 10.1039/d0nj02592e

rsc.li/njc

\title{
Interpretable machine learning as a tool for scientific discovery in chemistry
}

\author{
Richard Dybowski (D) *
}

\begin{abstract}
There has been an upsurge of interest in applying machine-learning $(\mathrm{ML})$ techniques to chemistry, and a number of these applications have achieved impressive predictive accuracies; however, they have done so without providing any insight into what has been learnt from the training data. The interpretation of ML systems (i.e., a statement of what an ML system has learnt from data) is still in its infancy, but interpretation can lead to scientific discovery, and examples of this are given in the areas of drug discovery and quantum chemistry. It is proposed that a research programme be designed that systematically compares the various model-agnostic and model-specific approaches to interpretable ML within a range of chemical scenarios.
\end{abstract}

\section{$1 \mathrm{Al}$ and $\mathrm{ML}$}

Artificial intelligence (AI) is a large and complex subfield of computer science concerned with the development of algorithms that mimic (to some degree) human cognitive functions such as learning, image recognition and natural language processing. ${ }^{1}$ It is believed that induction (generalising from finite examples) is an innate cognitive attribute, ${ }^{2}$ and induction is the core interest of machine learning (ML), ${ }^{3}$ which has become a prominent part of AI.

Numerous techniques have been developed under the heading of ML including neural networks, support vector machines and random forests, ${ }^{4}$ but, in line with the concept of 'statistical learning, ${ }^{5}$ we will also regard all forms of statistical regression as being under the ML umbrella.

\subsection{Deep learning}

At the heart of neural-based computation is the concept of an artificial neural network. ${ }^{6}$ The term "deep neural networks" (DNNs) refers to neural networks that have several internal layers of neurons (Fig. 1), and their strength lies in their ability to make multiple non-linear transformations through these layers of neurons. ${ }^{7}$ In this process, increasingly complex and abstract features can be constructed by the addition of more layers and/or increasing the number of neurons per layer. Each layer can be thought of as performing an abstraction of the information held within the preceding layer, so that a sequence of layers provides a hierarchy of increasing abstraction. This can obviate the need for manual selection of input features.

St John's College, University of Cambridge, Cambridge CB2 1TP, UK.

E-mail:rd460@cam.ac.uk

\subsection{Convolutional neural networks}

Convolutional neural networks (CNNs) are a subclass of DNNs. $\mathrm{CNNs}^{8}$ are inspired by the Hubel-Wiesel model of the visual primary cortex ${ }^{9}$ in which complex images are built up from simple features. The standard architecture of a CNN consists of alternating convolutional and pooling layers. A convolutional layer preserves the relationship between values in a matrix by multiplying a submatrix of the layer with a matrix filter to produce a 'feature map'. As a result, the network learns filters that activate when it detects some specific type of feature at some spatial position in the input.

A pooling layer abstracts the values of a feature map. This successive use of convolutional and pooling layers produces a hierarchy of abstracted features from an image that are invariant to translation, hence the successful use of CNNs for the recognition of images such as faces.

\section{$2 M L$ for chemistry}

ML techniques, such as deep neural networks, have become an indispensable tool for a wide range of applications such as image

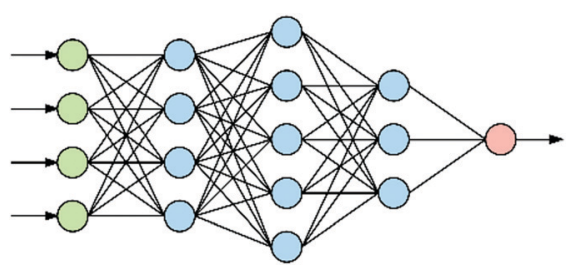

Fig. 1 An artificial neural network with three hidden layers (blue) between the input (green) and output (red) nodes: a deep neural network. Note that there can be more that 4 input nodes as well as more than one output node and many hidden layers. 
classification, speech recognition, and natural language processing. These techniques have achieved extremely high predictive accuracy, in many cases, on par with human performance.

There are many examples of where ML has been applied within chemistry, ${ }^{10}$ including the design of crystalline structures, ${ }^{11}$ planning retrosynthesis routes, ${ }^{12}$ and reaction optimisation. ${ }^{13}$ Here, we will briefly look at two; namely, drug discovery and quantum chemistry.

\subsection{Drug discovery}

The purpose of drug discovery is to find a compound that will dock with a biomolecule believed to be associated with a disease of interest (Fig. 2).

Once a target biomolecule (usually a protein) that is associated with a disease enters a pharmaceutical company's pipeline, it can take about 12 years to develop a marketable drug, but the failure rate during this process is high and costly. Each new drug that does reach the market represents research and development costs of close to one billion US dollars; therefore, early drug validation is vital, and this has led to the rise of computational (in silico) techniques. ${ }^{14}$ Consequently, the traditional techniques for drug discovery and target validation ${ }^{15,16}$ have been augmented with the use of machine learning to reduce the number of candidates by predicting whether a chemical substance will have activity at a given target. ${ }^{17}$ An example is the use of an ANN to aid the design of anti-bladder cancer agents. ${ }^{18}$

The standard approach to developing a neural network to predict whether a compound $S$ will be active with respect to a target protein $P$ is to train the network using a collection $\left\{S_{1}, \ldots, S_{n}\right\}$ of compounds with known activity toward $P$, but how can a ligand-protein activity predictor be trained if there are no known ligands for target protein $P$ ? AtomNet ${ }^{19}$ is a CNN designed to predict ligand-protein activities when no ligand activity for a target protein is available. This is done by training the CNN using known activities across a range of ligandprotein complexes. The thesis of AtomNet is as follows: (i) a complex ligand-protein interaction can be viewed as a combination of smaller and smaller pieces of chemical information; (ii) a CNN can model hierarchical combinations of simpler items of information; (iii) therefore, a CNN can model complex ligand-protein interactions.

Ligand-protein associations were encoded for AtomNet using ligand-protein interaction Fingerprints. The network significantly outperformed a variant of AutoDock Vina; ${ }^{20}$ for example, AtomNet achieved an AUC (Area Under ROC Curve)

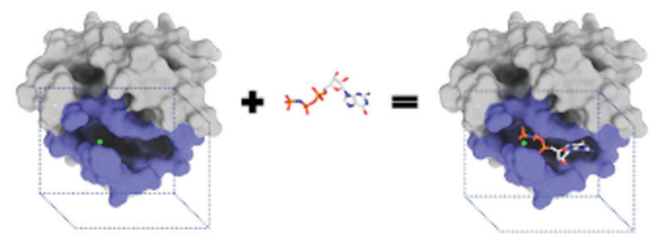

Fig. 2 A docking of guanosine-5'-triphosphate with the H-Ras p21 protein. greater than 0.9 for $57.8 \%$ of the targets in the DUDE dataset (Directory of Useful Decoys [false positives]). ${ }^{21}$

It is important when using ML for drug discovery that the ligand-protein data used to test the ML system is not biased. ${ }^{22}$

\subsection{Quantum chemistry}

The eigenvalue of the electronic Schrödinger equation $H \Psi=E \Psi$ gives total energy $E$, were $H$ is the Hamiltonian operator and $\Psi$ the wave function, but solving the equation analytically is limited to a few very simple cases. Therefore, for larger molecules, numerical approximation has been proposed, but with these methods, increasing accuracy is achieved at the cost of increasing run time (Table 1). Consequently, turning to ML was done to see if this enables $E$ to be estimated for larger molecules in radically shorter times without loss of accuracy (Fig. 3).

The set of nuclear charges $\left\{Z_{i}\right\}$ and atomic Cartesian coordinates $\left\{R_{i}\right\}$ uniquely determine the Hamiltonian $H$ of any chemical compound,

$$
H(\{Z, R\}) \stackrel{\Psi}{\mapsto} E,
$$

but is it possible to replace the use of $H$ with ML:

$$
\{Z, R\} \stackrel{\mathrm{ML}}{\mapsto} E ?
$$

An example of the use of ML for quantum chemistry is the mapping of $\{Z, R\}$ to $E$ by encoding the information in $\{Z, R\}$ using a Coulomb matrix $M:^{25}$

$$
M_{i, j}=\left\{\begin{array}{cl}
0.5 Z_{i}^{2.5}, & \text { if } i=j \\
\frac{Z_{i} Z_{j}}{\left|R_{i}-R_{j}\right|}, & \text { if } i \neq j .
\end{array}\right.
$$

Off-diagonal elements correspond to the Coulomb repulsion between atoms $i$ and $j$, while diagonal elements encode a polynomial fit of atomic energies to nuclear charge. The view of the authors was that the Coulomb matrix $M$ appropriately represents the required physics of the domain.

The data set $\left\{M_{k}, E_{k}^{\text {ref }}\right\}$ consisted of 7165 organic compounds, encoded as Coulomb matrices $M$, along with their energies $E^{\text {ref }}$ calculated using the PBE0 density function model. Cross-validation gave a mean absolute prediction error of $9.9 \mathrm{kcal} \mathrm{mol}^{-1}$.

Table 1 Hierarchy of numerical approximations to Schrödinger's equation. $N=$ system size s3 $^{23}$

\begin{tabular}{ll}
\hline Method & Runtime \\
\hline Configuration interaction (up to quadruple excitations) & $O\left(N^{10}\right)$ \\
Coupled cluster (CCSD(T)) & $O\left(N^{7}\right)$ \\
Configuration interaction (single and double excitations) & $O\left(N^{6}\right)$ \\
Møller-Plesset second-order perturbation theory & $O\left(N^{5}\right)$ \\
Hartree-Fock & $O\left(N^{4}\right)$ \\
Density functional theory (Kohn-Sham) & $O\left(N^{3-4}\right)$ \\
Tight binding & $O\left(N^{3}\right)$ \\
Molecular mechanics & $O\left(N^{2}\right)$
\end{tabular}




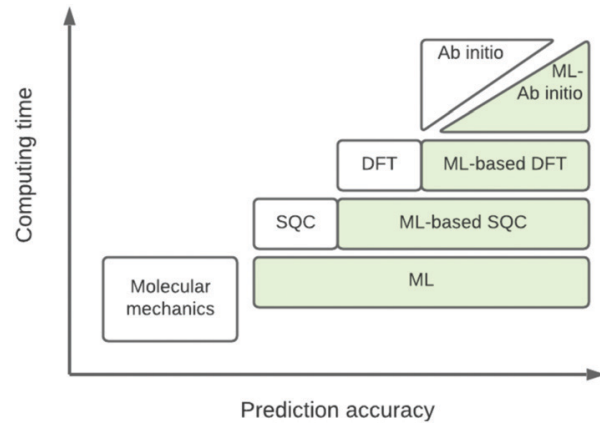

Fig. 3 Schematic representation of quantum chemical and ML approximations with respect to computational cost and accuracy, which generalises the literature. ${ }^{24}$ DFT $=$ density functional theory; $S Q C=$ semi-quantitative quantum chemistry.

\section{Scientific discovery}

The examples shown in the previous section focused on the use of ML for prediction, but what about scientific insight? Neural networks are so-called 'black-box' systems, meaning that the mapping of a vector of input values to a neural network's output is too computationally complex for a human to comprehend; therefore, how can we, for example, discover what chemistry the AtomNet CNN has learnt from the vast amount of ligand-protein-interaction data used to train it for in silico drug screening?

The idea of using AI for scientific discovery is not new, ${ }^{26}$ and there has recently been interest in using ML to provide scientific insights as well as making accurate predictions. ${ }^{27}$

\subsection{Interpretable ML}

Currently, an important requirement of AI systems is not only the accuracy of the conclusions reached by the systems but also transparency as to how the conclusions were reached. Algorithms, particularly ML algorithms, are increasingly important to peoples' lives, but they have caused a range of concerns revolving mainly around unfairness, discrimination and opacity. This has led to a "right to an explanation" under the EU General Data Protection Regulation.

Interpretability is an ill-defined concept,$^{28}$ but it will suffice for us to use the definition that interpretable ML is the use of ML models for the extraction of relevant knowledge about domain relationships contained in data. ${ }^{29}$ Consequently, interpretability refers to the extent to which a human expert can comprehend what an ML system has learnt from data; for example, "What is this ML system telling us?" From an interpretation we have insight, and from insight we can hopefully make a scientific discovery.

There are several categories of interpretability in the context of ML. In the following list, $f(\mathbf{x})$ will be written as $f(\mathbf{x} ; \hat{\boldsymbol{\Theta}})$, where $\hat{\boldsymbol{\Theta}}$ is the set of ML parameters estimated from training data.

(a) Observe the input-output behaviour of $f(\mathbf{x} ; \hat{\boldsymbol{\Theta}})$; for example, by observing how $f(\mathbf{x} ; \hat{\boldsymbol{\Theta}})$ varies as $\mathbf{x}$ is varied. (b) Inspect the values of parameters $\hat{\boldsymbol{\Theta}}$ within the internal structure of $f(\mathbf{x} ; \hat{\boldsymbol{\Theta}})$. Here, $f(\mathbf{x} ; \hat{\boldsymbol{\Theta}})$ is either intrinsically interpretable or is interpretable by design. This allows mapping $\mathbf{x} \rightarrow$ $f(\mathbf{x} ; \hat{\boldsymbol{\Theta}})$ to be understood by a series of steps going from input $\mathbf{x}$ to output $f(\mathbf{x} ; \hat{\boldsymbol{\Theta}})$ that are comprehensible to a domain expert. This can be regarded as an 'explanation' of how $f(\mathbf{x} ; \hat{\boldsymbol{\Theta}})$ was derived from $\mathbf{x}$.

(c) Determine the prototypical value $\mathbf{x}$ of for a given specific value $f^{*}$ of $f(\mathbf{x} ; \hat{\boldsymbol{\Theta}})$. Conceptually, this can be regarded as the $\mathbf{x}$ that maximises conditional probability $p\left(\mathbf{x} \mid f^{*}\right)$. $\mathbf{x}$ need not have been previously encountered in a training set. An example of this approach is activation maximization. ${ }^{30}$

A simple example of an intrinsically interpretable ML system is a linear regression model

$$
\widehat{\mathbb{E}}\left[y \mid x_{1}, \ldots, x_{n}\right]=\beta_{0}+\sum_{i=1}^{n} \beta_{i} x_{i}
$$

where $\beta_{0}, \beta_{n}$ are regression coefficients, and another is a decision tree induced from data. ${ }^{31}$

The potential of using interpretable ML for chemistry is starting to grow. For example, Bayesian neural networks have been optimised to predict the dissociation time of the unmethylated and tetramethylated 1,2-dioxetane molecules from only the initial nuclear geometries and velocities. ${ }^{32}$ Conceptual information was extracted from the large amount of data produced by simulations.

We now look at two other examples of interpretable ML: one from drug discovery; the other from quantum chemistry.

\subsection{Drug discovery and interpretability}

There are generally two approaches to providing interpretable ML: the model-agnostic and model-specific approaches (Fig. 4). Model-agnostic methods are, in principle, applicable to any black-box ML system $f(\mathbf{x})$, whereas the model-specific approach uses a domain-specific ML system, the structure of which is (at least partly) meaningful within the domain of interest.

There are two types of model-agnostic techniques. One method is the association-based technique in which associations are determined between inputs to system $f(\mathbf{x})$ and outputs from the system. One example of this are partial dependency plots, ${ }^{5}$ which create sets of ordered pairs $\left\{\left(\mathbf{x}_{s}^{(i)}, f\left(\mathbf{x}_{s}^{(i)}\right)\right\}\right.$ where feature subset $\mathbf{x}_{s} \subset \mathbf{x}$. Another way to examine how $f(\mathbf{x})$ changes as $x_{j}\left(x_{j} \in \mathbf{x}\right)$ changes is to use the 'gradient input' $x_{i}^{*} \partial f(\mathbf{x}) / \partial x_{i}$, where $x_{i}^{*}$ is a particular value of $x_{i}$. However, an extension of this is to integrate the gradient along a path for $x_{i}$ from observed value $x_{i}^{*}$ to a baseline value $x_{i}^{\prime}$. This is called an 'integrated gradient':

$$
\left.\left(x_{i}^{*}-x_{i}^{\prime}\right) \int_{\alpha=0}^{1} \frac{\partial f(\tilde{\mathbf{x}})}{\partial \tilde{x}_{i}}\right|_{\tilde{x}=x^{\prime}+\alpha\left(x_{i}^{*}-x_{i}^{\prime}\right)} \mathrm{d} \alpha .
$$

Integrated gradient $\mathrm{s}^{33}$ determined the chemical substructures (toxicophores) that are important for differentiating toxic and non-toxic compounds. The relevant substructures identified in 


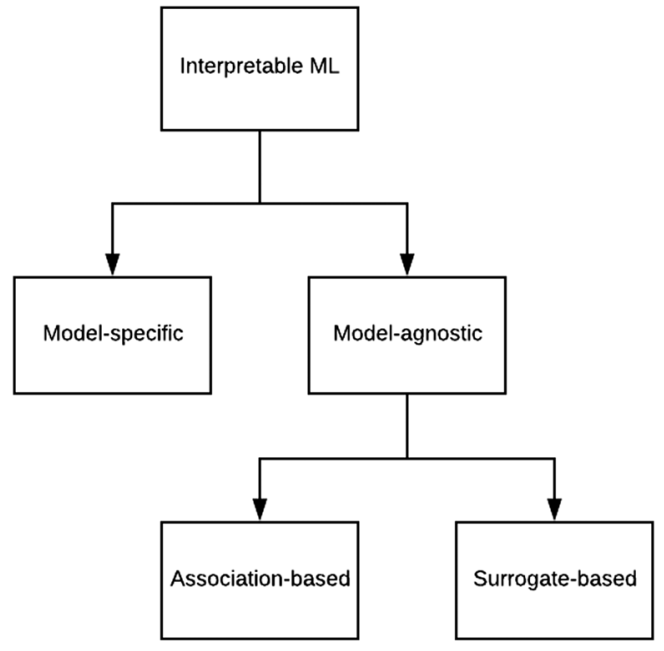

Fig. 4 Types of interpretable ML.

12 compounds randomly sampled from the Tox 21 Challenge data set are shown in Fig. 5. The DNN consisted of four hidden layers, each with 2048 nodes. The molecular structures were encoded using ECFPs, the training and test sets had 12060 and 647 examples, respectively, and the resulting AUC was 0.78.

An alternative to the detection of features relevant to a classification performed by a DNN is to start at an output node and work back to the input nodes. This is done with Layer-Wise Relevance Propagation (LRP), ${ }^{34}$ which uses the network weights and the neural activations of a DNN to propagate the output (at layer $M$ ) back through the network up until the input layer (layer 1). The backward pass is a conservative relevance

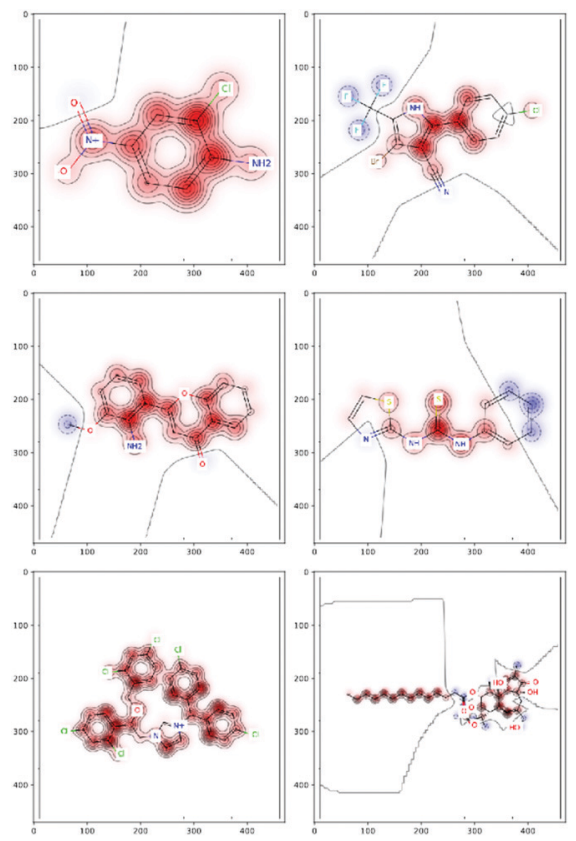

Fig. 5 Six randomly drawn Tox21 samples. Dark red indicates that these atoms are responsible for a positive classification, whereas dark blue atoms attribute to a negative classification. ${ }^{33}$ redistribution procedure where those neurons in layer $l$ $(1 \leq l<M)$ that contribute the most to layer $l+1$ receive the most 'relevance' from it. LRP has, so far, only been used to detect relevant features in pixel-based images and has not been used for the interpretation of chemistry-oriented ML systems. For example, rather than use fingerprints, such as ECPF, for molecular structure input, 2D molecular drawings have been used as inputs to a CNN (and achieved a predictive accuracy of AUC 0.766$)^{35}$ but LRP was not used for interpretation.

Graph convolutional neural networks (GCNNs) are a variant of CNNs that enable 3D graphs to be used as inputs. Consequently, if the nuclei and bonds of a compound are regarded as the vertices and edges of a 3D graph then 3D molecular structures can be considered as inputs to GCNNs. One approach is to initially slide convolutional filters over atom pairs to obtain atom-pair representations; ${ }^{33}$ pooling is then used to produce simple substructure representations. These representations were then fed into the next convolutional layer. The predictive accuracy of the resulting GCNN was an AUC of 0.714. Interpretation was done by omitting the pooling steps and feeding the substructures directly into the fully connected network.

The other type of model-agnostic approach is the use of surrogates (Fig. 6); namely, using a function $\tilde{f}(\mathbf{x})$ that is an approximation of black box $f(\mathbf{x})$ but which is intrinsically interpretable. Examples of parsimonious intrinsically interpretable models include linear regression, logistic regression and decision trees. Such models can be either global or local. Given a set of vectors $\left\{\mathbf{x}^{(1)}, \ldots, \mathbf{x}^{(n)}\right\}$ nd that we wish to apply to $f(\mathbf{x})$, the global approach is to apply each of these vectors to the same surrogate model $\tilde{f}(\mathbf{x})$. In contrast, the local approach uses a different surrogate model $\tilde{f}_{i}\left(\mathbf{x}^{(i)}\right)$ for vector $\mathbf{x}^{(i)}$. An example is the LIME technique, ${ }^{36}$ which trains $\tilde{f}_{i}\left(\mathbf{x}^{(i)}\right)$ on data in the "neighbourhood' of $\mathbf{x}^{(i)}$, thereby providing interpretability specifically for the input-output pair $\left(\mathbf{x}^{(i)}, \tilde{f}_{i}\left(\mathbf{x}^{(i)}\right)\right)$.

\subsection{Quantum chemistry and interpretability}

The above perspectives to interpreting a neural network focus on discovering associations between values at the input and output nodes: values at the hidden nodes are ignored. In contrast, another way of attempting to produce interpretable neural networks is to use internal nodes that are meaningful with respect to a domain of interest. This idea is not new and is at the heart of neuro-fuzzy systems ${ }^{37}$ in which the interpretability of a neural network is provided by chains of inference via fuzzy logic. ${ }^{38}$

(a)

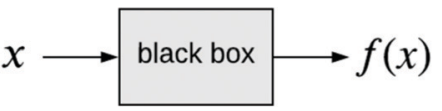

(b)

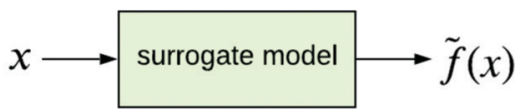

Fig. 6 (a) Black box trained from data $\{x, y\}$. (b) Surrogate model of the black box trained from the same data. $\tilde{f}(\mathbf{x})$ approximates $f(\mathbf{x})$. 


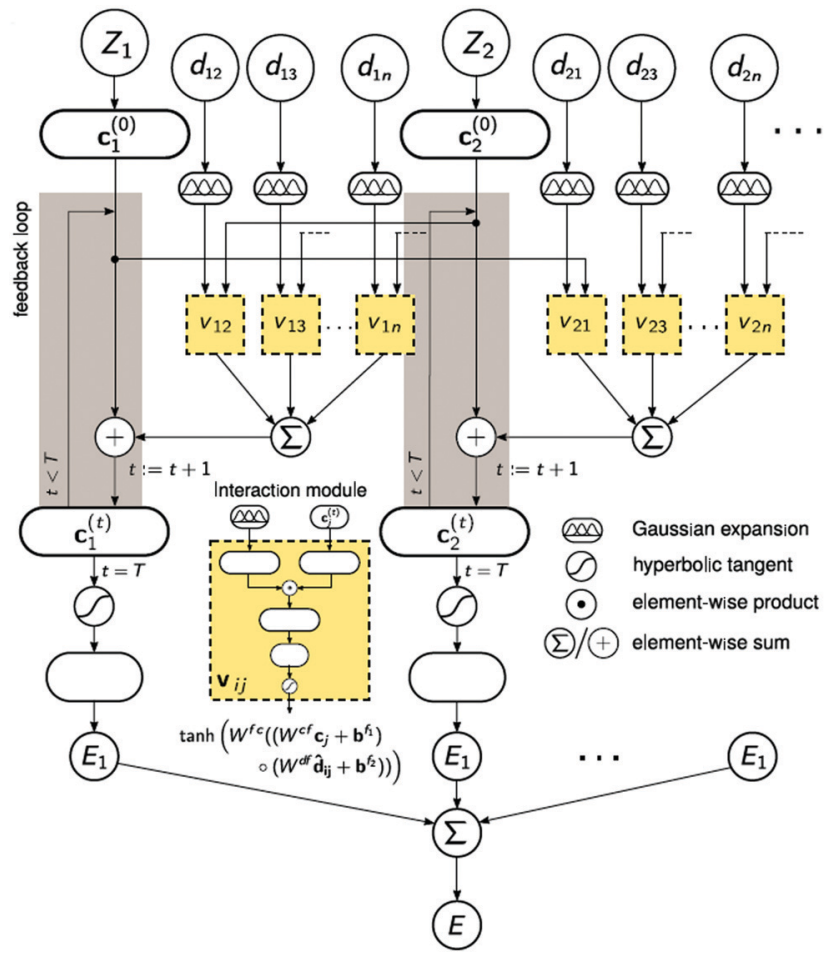

Fig. 7 The architecture of SchNet. ${ }^{39}$ The iteration loop implements eqn (1), and the interaction module (a neural network) implements eqn (2).

Rather than resorting to fuzzy logic, neural networks such as SchNet (described below) are constructed by combining science-based subsystems in a plausible manner. This is an example of the model-specific approach to interpretable ML (Fig. 4).

A strategy for molecular energy $E$ prediction $^{39}$ is to represent each atom $i$ by a vector $\mathbf{c}_{i}$ in B-dimensional space, and a deep tensor neural network (DTNN) called SchNet, shown in Fig. 7, repeatedly refines $\mathbf{c}_{i}$ by pair-wise interaction between atoms $i$ and $j$ from an initial vector $\mathbf{c}_{i}^{(0)}$ for atom $i$ to final vector $\mathbf{c}_{i}^{(T)}$ :

$$
\mathbf{c}_{i}^{(t+1)}=\mathbf{c}_{i}^{(t)}+\sum_{j \neq i} \mathbf{v}_{i j}
$$

Interaction term $\mathbf{v}_{i j}$ reflects the influence of atom $j$ at a distance $\mathbf{d}_{i j}$ on atom $i$ (the amount of overlap), and each refinement step aims to reduce these overlaps. For the interactions, the distances between atoms are expanded in a Gaussian basis.

Term $\mathbf{v}_{i j}$ is obtained from atom vector $\mathbf{c}_{j}$ and distance $\mathbf{d}_{i j}$ using a feedforward neural network with a tanh activation function:

$$
\mathbf{v}_{i j}=\tanh \left[W^{f c}\left(W^{c f} \mathbf{c}_{j}+\mathbf{b}^{f 1}\right) \bigcirc\left(W^{d f} \mathbf{d}_{i j}+\mathbf{b}^{f 2}\right)\right]
$$

where $W^{c f}, \mathbf{b}^{f 1}, W^{d f}, \mathbf{b}^{f 2}$, and $W^{c f}$ are the weight matrices and corresponding biases of atom representations, distances and resulting factors, respectively.

After $T$ iterations, an energy contribution $E_{i}$ for atom $i$ is predicted for the final vector $\mathbf{c}_{i}^{(T)}$, and the total energy $E$ is the sum of the predicted contributions $E_{i}$.

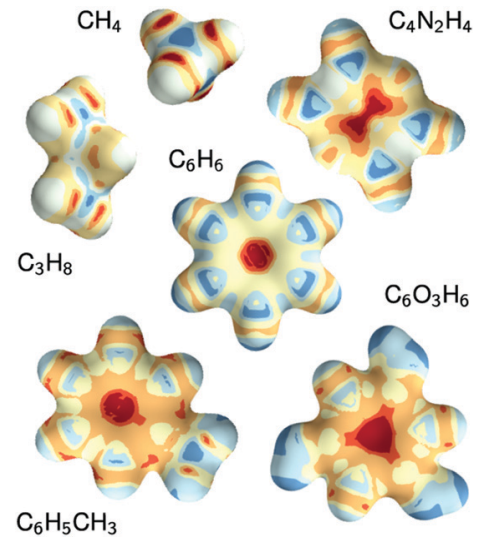

Fig. 8 Chemical potentials for methane, propane, pyrazine, benzene, toluene, and phloroglucinol determined from SchNet. ${ }^{39}$

The DTNN, trained using stochastic gradient descent, achieved a mean absolute error of $1.0 \mathrm{kcal} \mathrm{mol}^{-1}$ on the GDB datasets.

The constructive nature of the DTNN allows interpretation of how $E$ is obtained, and the estimation of $E$ allows energy isosurfaces to be constructed (Fig. 8).

Returning to the Schrödinger equation (in Dirac notation),

$$
H\left|\Psi_{n}\right\rangle=E\left|\Psi_{n}\right\rangle
$$

The Hartree-Fock method for molecular orbitals is to approximate a wave function $|\Psi\rangle$ for a molecular orbital as a linear combination of atomic orbitals:

$$
\left|\Psi_{n}\right\rangle=\sum_{i=1}^{N} k_{n, i}\left|\phi_{i}\right\rangle
$$

where $\left\{\left|\phi_{i}\right\rangle\right\}$ is a set of $N$ basis functions, and $\left\{k_{i}\right\}$ are the associated coefficients. Function $\left|\phi_{i}\right\rangle$ can be an atom-centred Gaussian function. As a consequence, the electronic Schrödinger can be written in the matrix form

$$
\mathbf{H k}_{m}=\varepsilon_{m} \mathbf{S} \mathbf{k}_{m}
$$

where the Hamiltonian matrix has elements

$$
H_{i, j}=\left\langle\phi_{i}|H| \phi_{j}\right\rangle
$$

and the overlap matrix has elements

$$
S_{i, j}=\left\langle\phi_{i} \mid \phi_{j}\right\rangle
$$

where $S_{i, j}$ measures the extent to which two basis functions overlap.

SchNOrb $^{40}$ was developed to predict $\mathbf{H}$ and $\mathbf{S}$ using ML. The first part of the structure of SchNOrb is identical to SchNet in that it starts from initial representations of atom types and positions, continues with the construction of representations of chemical environments of atoms and atom pairs (again identical to the method used in SchNet) but then uses these to predict energy $E$ and Hamiltonian matrix $\mathbf{H}$, respectively.

The SchNet and SchNOrb systems illustrate how DNNs can be customized to specific scientific applications so that the DNN architecture promotes properties that are desirable in the 
data modelled by the network. Interpretation occurs by unpacking these networks. This strategy has already found success in several scientific areas including plasma physics ${ }^{41}$ and epidemiology. ${ }^{42}$ Another example is the use of a customised $\mathrm{DNN}^{43}$ to encode the hierarchical structure of a gene ontology to provide insight into the structure and function of a cell.

\section{Discussion}

We have briefly looked at the types of interpretable ML referred to in Fig. 4 and provided some chemical examples. In order to move forward, it is proposed that a research programme be designed that systematically compares the applicability and efficacy of the techniques over a wide range of chemical scenarios. The results of this exercise should be compiled into an on-line guide to support research (perhaps in an manner analogous to what was attempted for ML in the $1990 \mathrm{~s}^{44}$ ). One of the existing Internet platforms for digital research collaboration might suffice.

Roscher et al. ${ }^{27}$ divided the various forms of ML for scientific discovery into four groups. Group 1 includes approaches without any means of interpretability. With Group 2, a first level of interpretability is added by employing domain knowledge to design the models or explain the outcomes. Group 3 deals with specific tools included in the respective algorithms or applied to their outputs to make them interpretable, and Group 4 lists approaches where scientific insights are gained by explaining the machine learning model itself. These categories should help to structure the above proposed systematic comparison.

As regards the model-specific and model-agnostic approaches, it is anticipated that the agnostic method is more widely applicable because of the greater propensity of black-box systems.

The architectures of SchNet and SchNOrb are not learned but are designed with prior knowledge about the underlying physical process. In contrast, the aim of SciNet $^{45}$ is to learn, without prior scientific knowledge, the underlying physics of a system from combinations of (a) observations (experimental data) taken from the physical system, (b) questions asked about the system, and (c) the correct answers to those questions in the context of the observations. This is done by attempting to use the latent neurons of an autoencoder network ${ }^{3}$ to learn and represent underlying physical parameters of the system. A network's learned representation is interpreted by analysing how the region of latent neurons responds to changes in the values of known physical parameters. For example, when given a time series of the positions of the Sun and the Moon, as seen from Earth, SciNet deduced Copernicus' heliocentric model of the solar system. This course to scientific discovery has not yet been applied to chemical systems but, given its potential, it is suggested that a clear methodology be developed that extends the SciNet-type approach to help chemists uncover new ideas and the links between them.

The exploration of the potential of interpretable ML to the sciences is growing, with applications in genomics, ${ }^{46}$ manybody systems, ${ }^{47}$ neuroscience ${ }^{48}$ and chemistry. Although this chemical review has focused on interpretation with respect to drug discovery and quantum chemistry, the potential of ML has been explored in other areas of chemistry, such as the use of ML for computational heterogeneous catalysis ${ }^{49}$ and retrosynthesis, ${ }^{50}$ and the use of interpretable ML in these and other fields is expected to prove to be immensely useful.

But a cautionary note. Reproducibility is fundamental to scientific research; thus, it is crucial that scientific discoveries arising from ML are reproducible, and this need must be factored into any methodology built for ML-based discovery. And the provision of raw research data with a publication is essential to overcome the "reproducibility crises". 51

In February 2020, the Alan Turing Institute held a workshop that announced the Nobel Turing Challenge; namely, "the production of AI systems capable of making Nobel-quality scientific discoveries highly autonomously at a level comparable, and possibly superior, to the best human scientists by 2050". Chemistry is within the remit of the Challenge, and it is anticipated that interpretable ML will play a vital role toward the production of an 'AI Chemist'.

\section{Conflicts of interest}

There are no conflicts to declare.

\section{Acknowledgements}

We thank Pavlo Dral (Xiamen University) for discussions regarding Fig. 3, and Laurent Dardenne (DockThor), Thomas Unterthiner (Johannes Kepler University) and Alexandre Tkatchenko (University of Luxembourg) for permission to reproduce Fig. 2, 5, 7 and 8. We thank the Department of Chemistry, Cambridge University, for the Visiting Researchship. This work is dedicated to Zofia Kaczan-Aniecko.

\section{Notes and references}

1 S. Russell and P. Norvig, Artificial Intelligence: A Modern Approach, Pearson, Boston, 2010.

2 Induction: Processe of Inference, Learning and Discovery, ed. J. Holland, K. Holyoak, R. Nisbett and P. Thagard, MIT Press, Cambridge, MA, 1986.

3 I. Goodfellow, Y. Bengio and A. Courville, Deep Learning, MIT Press, Cambridge, MA, 2016.

4 K. Murphy, Machine Learning: A Probabilistic Perspective, MIT Press, 2012.

5 T. Hastie, R. Tibshirani and J. Friedman, The Elements of Statistical Learning, Springer, New York, 2nd edn, 2008.

6 C. Bishop, Neural Networks for Pattern Recognition, Clarendon Press, Oxford, 1995.

7 G. Goh, N. Hodas and A. Vishnu, 2017, arXiv, 1701.04503.

8 Y. LeCun, Cognitiva, 1985, 85, 599-604.

9 D. Hubel and T. Wiesel, J. Physiol., 1962, 160, 106-154.

10 T. Cova and A. Pais, Front. Chem., 2019, 7, 809.

11 T. Xie and J. Grossman, 2017, arXiv, 1710.10324. 
12 M. Segler, M. Preuss and M. Waller, Nature, 2018, 555, 604-610.

13 Z. Zhou, X. Li and R. Zare, ACS Cent. Sci., 2017, 3, 1337-1344.

14 S. Smith, Nature, 2003, 422, 341-347.

15 M. Lindsay, Nat. Rev. Drug Discovery, 2003, 2, 831-838.

16 S. Wang, T. Sim, Y.-S. Kim and Y.-T. Chang, Curr. Opin. Chem. Biol., 2004, 8, 371-377.

17 A. Lavecchia, Drug Discovery Today, 2015, 20, 318-331.

18 A. Speck-Planche, V. Kleandrova, F. Luan and M. Cordeiro, Anticancer Agents Med. Chem., 2013, 13, 791-800.

19 I. Wallach, M. Dzamba and A. Heifets, 2015, ArXiv, 1510.02855.

20 O. Trott and A. Olson, J. Comput. Chem., 2010, 31, 455-461.

21 M. Mysinger, M. Carchia, J. J. Irwin and B. Shoichet, J. Med. Chem., 2012, 55, 6582-6594.

22 L. Chen, A. Cruz, S. Ramsey, C. Dickson, J. Duca, V. Hornak, D. Koes and T. Kurtzman, PLoS One, 2019, 14, e0220113.

23 T. Unterthiner, A. Mayr, G. Klambauer, M. Steijaert, J. Wegner, H. Ceulemans and S. Hochreiter, Deep Learning as an Opportunity in Virtual Screening, 2014, NIPS Workshop on Deep Learning and Representation Learning, Montreal, 12 December 2014.

24 P. Dral, J. Phys. Chem. Lett., 2020, 11, 2336-2347.

25 M. Rupp, A. Tkatchenko, K.-R. Muller and O. A. von Lilienfeld, 2011, ArXiv, 1109.2618.

26 P. Langley, H. Simon, G. Bradshaw and J. Zytkow, Scientific Discovery, MIT Press, Cambridge, MA, 1987.

27 R. Roscher, B. B. M. Duarte and J. Garcke, 2019, ArXiv, 1905.08883v2.

28 L. Gilpin, D. Bau, B. Yuan, A. Bajwa, M. Specter and L. Kagal, 2019, ArXiv, 1806.00096v3.

29 W. Murdoch, C. Singh, K. Kumbier, R. Abbasi-Asl and B. Yu, 2019, ArXiv, 1901.04592.

30 A. Mahendran and A. Vedaldi, 2016, arXiv, 1512.02017.

31 L. Breiman, J. Friedman, R. Olshen and C. Stone, Classification and Regression Trees, Chapman \& Hall, New York, 1984.

32 F. Häse, I. Galván and M. Vache, Chem. Sci., 2019, 10, 2298.
33 K. Preuer, G. Klambauer, F. Rippmann, S. Hochreiter and T. Unterthiner, 2019, ArXiv, 1903.02788v2.

34 S. Bach, A. Binder, G. Montavon, F. Klauschen, K.-R. Muller and W. Samek, PLoS One, 2015, 10, e0130140.

35 G. Goh, C. Siegel, A. Vishnu, N. Hodas and N. Baker, 2017, ArXiv, 1706.06689.

36 S. Singh, M. Ribeiro and C. Guestrin, 2016, arXiv, 1602.04938.

37 D. Nauck, F. Klawonn and R. Kruse, Foundations of NeuroFuzzy Systems, Wiley, Chichester, 1997.

38 L. Zadeh, Fuzzy Sets Syst., 1978, 1, 3-28.

39 K. Schütt, F. Arbabzadah, S. Chmiela, K. Müller and A. Tkatchenko, 2016, ArXiv, 1609.08259v3.

40 K. Schütt, M. Gastegger, A. Tkatchenko, K.-R. Müller and R. Maurer, Nat. Commun., 2019, 10, 5024.

41 F. Matos, F. Hendrich, F. Jenko and T. Odstrcil, 82nd Annual Meeting of the DPG and DPG Spring Meeting of the AMOP Section, Friedrich Alexander University of Erlangen-Nuremberg, 2018.

42 A. Adiga, C. Kuhlman, M. Marathe, H. Mortveit, S. Ravi and A. Vullikanti, Int. J. Adv. Eng. Sci. Appl. Math., 2018, 11, 153-171. 43 J. Ma, M. Yu, S. Fong, K. Ono, E. Sage, B. Demchak, R. Sharan and T. Ideker, Nat. Methods, 2018, 15, 290-298.

44 Machine Learning: Neural and Statistical Classification, ed. D. Michie, D. Spiegelhalter and C. Taylor, Ellis Horwood, New York, 1994.

45 R. Iten, T. Metger, H. Wilming, L. Rio and R. Renner, 2018, ArXiv, 1807.10300v2.

46 D. Sharma, A. Durand, M.-A. Legault, L.-P. L. Perreault, A. Lemaon, M.-P. Dub and J. Pineau, 2020, arXiv, 2007.01516.

47 W. Zhong, J. Gold, S. Marzen, J. England and N. Halpern, 2020, arXiv, 2001.03623.

48 A. Balwani and E. Dyer, bioRxiv, 2020, DOI: 10.1101/ 2020.05.26.117473.

49 A. Peterson, J. Chem. Phys., 2016, 145, 074106.

50 M. Segler and M. Waller, Chem. - Eur. J., 2017, 23, 6118-6128.

51 T. Miyakawa, Mol. Brain, 2020, 13, 24. 